\title{
Activity of a novel, multimodal, silver-based skin cleanser on coronaviruses, in-vitro
}

\author{
Daryl S Paulson* \\ BioScience Laboratories, Inc., 1765 South 19th Avenue; Bozeman, MT 59718, USA
}

\begin{abstract}
Background: The primary objective of these tests was to evaluate the efficacy of a novel, multi-ingredient colloidal silver skin cleanser (Theraworx Protect, Avadim Technologies, Asheville, NC) on a seasonal coronavirus as a proxy for SARS-CoV-2 and 7 additional challenge microorganisms in vitro.

Methods: The coronavirus test measured percent and $\log 10$ reductions from the initial population of the viral strain(s) following exposure to the test product(s) at 1 minute, 30 minutes, and 1 hour. Plating was performed in four replicates.

The challenge microorganisms test used an In-Vitro Time-Kill Method to assess the antimicrobial properties of the test product and measured percent and log 10 reduction in the microbial population of each challenge strain following exposure to each test material for 60 seconds, 10 minutes, and 30 minutes. Testing was performed in triplicate. All agar-plating was performed in duplicate.

Results: At 1 and 30 minutes, there was a $1.75 \log$ reduction (98.22\%) in SARS-CoV-2 virus. At 60 minutes, there was a $2.25 \log$ reduction (99.44\%) in virus.

The test product, Theraworx Protect (Lot \#16180-1), reduced the populations of 7 challenge microorganisms - multidrug-resistant (MDR) Acinetobacter baumannii (ATCC\#BAA-1605), Hemophilus influenzae (ATCC\#19418), Pseudomonas aeruginosa (ATCC \#15442), Staphylococcus epidermidis (ATCC \#12228), Staphylococcus hominis (ATCC \#700236), Streptococcus pneumoniae (ATCC \#49619), Streptococcus pyogenes (ATCC \#19615) - by an average of greater than 3.4 log10 following 1 minute and maintained or increased these reductions through all the remaining appropriate time points.
\end{abstract}

Conclusion: The results confirm the test product's efficacy as a waterless, leave-on, skin-compatible, topical solution to augment the use of flashing ethanol-based hand sanitizers.

\section{Background}

Interventions to prevent and control infection are critical to reduce morbidity and mortality. Such interventions are often multimodal, with many different interventions in place for attacking transmission from various angles. This is because although each intervention (hand hygiene, social distancing, enhanced testing, quarantine, data sharing, media attention, etc.) is considered critical, none of them are foolproof. This model of safety incidents dates back 20 years in healthcare quality and improvement with James Reason naming it the "Swiss Cheese" model [1] In this paradigm, each intervention is like multiple pieces of swiss cheese, each slice having holes and unable to prevent something undesirable from one side reaching the other. No single slice (intervention) is perfect, so some undesirable consequences may pass through the holes of the cheese. However, if enough interventions are stacked, soon the holes are covered because not all interventions have the same faults or holes.

Autoinoculation of pathogens is a major mode of transmission for organisms capable of contact-type transmission. This pathway has historically considered only microbial contamination of the hands from environmental sources and instillation of those organisms into mucus membranes of the eyes, nose, or mouth. Currently, the severe acute respiratory syndrome coronavirus type 2 (SARS-CoV-2), the causative agent of coronavirus disease 2019 (COVID-19), is likely being transmitted via this autoinoculative pathway [2]. Because of this, hand hygiene is thought to be a critical prevention intervention for reducing the risk of transmission of this virus, and it is important to assess the activity of novel products on coronaviruses to identify potential adjunct or comparable modalities for intervention-similar to studies with SARS-CoV-1 [3].

The objective of this test was to evaluate the efficacy of a novel skin cleanser on a seasonal coronavirus as a proxy for SARS-CoV-2 and 7 additional challenge microorganisms: multidrug-resistant (MDR) Acinetobacter baumannii, Pseudomonas aeruginosa, Staphylococcus epidermidis, Staphylococcus hominis, Streptococcus pneumonia, Streptococcus pyogenes and Hemophilus influenzae.

\section{Material and methods}

The coronavirus study was designed to evaluate the virucidal properties of one test product [Theraworx Protect liquid, a novel, silver and surfactant-based, multimodal skin cleanser (Avadim Health; Asheville, NC)] against human coronavirus strain OC43 (ZeptoMetrix Corporation \#0810024CF) and 7 challenge microorganisms using a virucidal suspension test (in vitro time-kill method). The host cells were

${ }^{\star}$ Correspondence to: Daryl S Paulson, BioScience Laboratories, Inc., 1765 South 19th Avenue; Bozeman, MT 59718, USA, E-mail: DPaulson@biosciencelabs.com

Key words: coronavirus, hand sanitizer, colloidal silver, antimicrobial

Received: September 16, 2020; Accepted: September 25, 2020; Published: September 30, 2020 
HCT-8 (ATCC \#CCL-244; epithelial human colon adenocarcinoma). A third-party laboratory provided all laboratory testing (BioScience Laboratories, Inc.; Bozeman, MT), and testing was based on the standard ASTM E1052-11, Standard Test Method to Assess the Activity of Microbicides against Viruses in Suspension. All testing was performed in accordance with Good Laboratory Practice regulations, as specified in 21 CFR Part 58. The percent and $\log 10$ reductions from the initial population of the viral strain(s) were determined following exposure to the test product(s) at 1 minute, 30 minutes, and 1 hour. Plating was performed in four replicates.

The challenge microorganisms test used an In-Vitro Time-Kill Method to assess the antimicrobial properties of the test product and one reference product when challenged with suspensions of 16 microorganisms. This procedure was based upon methodology described in ASTM E2783-11, Standard Test Method for Assessment of Antimicrobial Activity for Water Miscible Compounds Using a Time-Kill Procedure. The percent and $\log 10$ reduction in the microbial population of each challenge strain was determined following exposure to each test material for 60 seconds, 10 minutes, and 30 minutes. Testing was performed in triplicate. All agar-plating was performed in duplicate.

\section{Results}

At 1 and 30 minutes, there was a $1.75 \log$ reduction (98.22\%) in SARS-CoV-2 virus. At 60 minutes, there was a 2.25 log reduction (99.44\%) in virus.

The test product, Theraworx Protect (Lot \#16180-1), reduced the populations of 7 challenge microorganisms - multidrug-resistant (MDR) Acinetobacter baumannii, (ATCC\#BAA-1605), Hemophilus influenzae (ATCC\#19418), Pseudomonas aeruginosa (ATCC \#15442), Staphylococcus epidermidis (ATCC \#12228), Staphylococcus hominis (ATCC \#700236), Streptococcus pneumoniae (ATCC \#49619), Streptococcus pyogenes (ATCC \#19615) - by an average of greater than $3.4 \log 10$ following 1 minute and maintained or increased these reductions through all the remaining appropriate time points.

\section{Discussion}

The CDC guidelines for healthcare-related hand hygiene recommend alcohol-based hand sanitizers with at least $62 \%$ alcohol [4]. For the non-healthcare setting, the CDC recommends soap and water for hand hygiene [5]. The test product employs specialized surfactant technology, making the innovation compliant with the CDC soap and water recommendation. The product also improves skin quality and has demonstrated six-hour duration of action equivalent to $4 \%$ chlorhexidine gluconate, ASTM Method E1173-15 simulated pre-operative skin preparation [6]. What's more, the test product has been evaluated against a broad array of microorganisms, including multidrug-resistant (MDR) Acinetobacter baumannii, Pseudomonas aeruginosa, Staphylococcus epidermidis, Staphylococcus hominis, Streptococcus pneumonia, Streptococcus pyogenes and Hemophilus influenzae, demonstrating a greater than $3.4 \log$ reduction after 1 minute.

Given the autoinoculative likelihood of various pathogens, including SARS-CoV-2, interventions such as this could be beneficial. Furthermore, this product has been shown to normalize the skin $\mathrm{pH} /$ acid mantle [7]. A critical aspect of maintaining skin barrier functions such as transient and resident flora regulation $[8,9]$.

\section{Conclusions}

We have tested a novel skin cleanser against a seasonal coronavirus (OC43)-which represents the same virus as COVID-19 and equivalent susceptibility patterns to antiseptics as would SARS-CoV-2, the causative agent of COVID-19-and found reasonable reduction in virus, particularly after an hour post-application. At 60 seconds, the product demonstrated a $1.75 \log$ reduction and greater than $2.0 \mathrm{log}$ reduction (or 99\%) at 60 minutes, demonstrating increasing efficacy with time. This novel product has greater persistence than alcohol, and it can be used as a persistent adjunct that is also safe to be used on mucus membranes including the eyes, nose and mouth (the main zone of transmission).

The results confirm the product's efficacy as a waterless, leaveon, skin-compatible, topical solution to augment the use of flashing ethanol-based hand sanitizers. Additionally, the results have shown this product's ability to hydrate and restore dermal tissue to a healthy state during the frequent use of traditional antiseptic actives, restoring the natural microflora as well as providing a persistent activity between ethanol product applications. Because of this, it may be possible that it could provide an extra layer of protection during the COVID-19 pandemic.

\section{References}

1. Reason James (2000) Human error: models and management. BMJ 320: 768 [Crossref]

2. Li Q, Guan X, Wu P, Wang X, Zhou L, et al. (2020) Early transmission dynamics in wuhan, china, of novel coronavirus-infected pneumonia. N Engl J Med 382: 1199- 207. [Crossref]

3. Rabenau HF, Kampf G, Cinatl J, Doerr HW (2005) Efficacy of various disinfectants against SARS coronavirus. $J$ Hosp Infect 61: 107-111. [Crossref]

4. CDC (2020) Hand hygiene in healthcare settings. Available at : https://www.cdc.gov/ handhygiene/providers/index.html.

5. CDC (2020) When and how to wash your hands. Available at: https://www.cdc.gov/ handwashing/when-how-handwashing.html. Accessed July 15, 2020

6. Paulson DS, Topp R, Boykin RE, Schultz G, Yang Q (2018) Efficacy and safety of a novel skin cleansing formulation versus chlorhexidine gluconate. Am J Infect Control 46: 1262-1265. [Crossref]

7. Woody S inventor (2016) Method for the prevention and treatment of acne. USA patent US $2016 / 0184220.2$

8. Larson E (1999) Skin hygiene and infection prevention: more of the same or different approaches?. Clin Infect Dis 29: 1287-1294. [Crossref]

9. Korting HC, Braun-Falco O (1996) The effect of detergents on skin pH and its consequences. Clin Dermatol 14: 23-27. [Crossref]

Copyright: (C2020 Paulson DS. This is an open-access article distributed under the terms of the Creative Commons Attribution License, which permits unrestricted use, distribution, and reproduction in any medium, provided the original author and source are credited. 